\title{
Correlated Surfaces of Free-Standing Polystyrene Thin Films
}

\author{
K. Shin, ${ }^{\dagger, \ddagger}$ Y. Pu, M. H. Rafailovich, ${ }^{*, \ddagger}$ J . Sokolov, ${ }^{\ddagger}$ O. H. Seeck, ${ }^{\S, \downarrow}$ S. K. Sinha, ${ }^{\perp}$ \\ M. Tolan, " and R. Kolb\$ \\ Department of Materials Science and Engineering, State University of New York, \\ Stony Brook, New York 11794-2275; XF D/ APS Argonne National Laboratory, \\ 9700 South Cass Avenue, Argonne, Illinois 60439; Institut für Experimentelle und Angewandte Physik \\ der Universität Kiel 24098 Kiel, Germany; and ExxonMobil Research and Engineering Company, \\ Annandale, New J ersey 08801
}

Received November 8, 2000; Revised Manuscript Received April 9, 2001

\begin{abstract}
We have performed X-ray specular and off-specular measurements of free-standing polystyrene thin films as a function of molecular weight and thickness. The results show films thicker than a few radii of gyration $\left(R_{g}\right)$ are well fit by a simple liquid model. This confirms the assumption that the anomalies previously reported in the scattering intensity from polymer films of comparable thickness were mostly due to confinement by long-ranged interactions with the substrate rather than a fundamental property of viscoelastic fluids. The simple liquid model was found to be insufficient to fit the transverse diffuse data for films thinner than a few $R_{\mathrm{g}}$. Longitudinal diffuse scattering data demonstrated that the roughness at the two interfaces is highly correlated when the film thicknesses are approximately $2 R_{g}$. Hence, very thin films do not exhibit liquidlike behavior, and higher-order elastic terms may have to be included into models to describe their rheological behavior.
\end{abstract}

\section{Introduction}

The bulk properties of polymers have been extensively studied. Recently, the importance of thin films has grown in both technological applications and scientific interest. 1,2 Industrial applications such as coatings, insulators, and adhesives to modify and enhance the surface properties motivate the research on polymer/ substrate or air interfaces. It was shown that for thin films on substrates the physical properties of the films such as mol ecular mobility, ${ }^{3,4}$ glass transitions, ${ }^{5,6}$ elasticity, and viscosity 7,8 could vary a great deal , compared to the corresponding polymer bulk properties. Proximity to an attractive substrate could also modify the properties of the free surface. For example, Wang et al. reported that the correlation function of the capillary wave fluctuations are dependent on the fil $\mathrm{m}$ thickness and substrate interaction. ${ }^{9}$ Frank et al. have reported a decrease in lateral chain diffusion, ${ }^{10}$ and Overney et al. have observed a decrease in friction and adhesion at the surface of supported thin films. ${ }^{11}$ The question as to whether these phenomena are fundamental to polymer thin films or whether they are due to pinning or effective cross-linking of the polymer layer adjacent to the substrate has not yet been answered. To address the fundamental issues regarding molecular dynamics in thin polymer films, we must first separate the influences of interactive surfaces from the internal structure basic to the film. Free-standing thin films, where the surfaces are not in contact with any substrate, can thus be used to investigate the finite size effects without being altered by substrates. ${ }^{12-16}$

\footnotetext{
* Corresponding author: E-mail mrafailovich@sunysb.edu. † Present address: NCNR, NIST, Gaithersburg, MD 208999562.

₹ SUNY at Stony Brook.

§ Present address: FZ J uelich GmbH, -IFF8-, 52425 J uelich, Germany.

${ }^{\perp}$ APS, ANL.

\#Universität Kiel.

$\$$ ExxonMobil RE \& C.
}

X-ray specular and diffuse scattering measurements are powerful tools to investigate the interfaces of thin films, including the basic physical properties, e.g., film thickness, roughness, surface tension, and conformality. ${ }^{17,18}$ In this paper we use specular reflectivity to determine roughnesses and film thicknesses of freestanding films. Longitudinal diffuse scattering is used to study the conformality between the roughnesses at the two interfaces. Transverse scattering is used to confirm that the capillary wave spectra from viscoelastic polymer films are identical to those of simple liquids. It has long been postulated that only local correlation should give rise to the capillary waves. ${ }^{17}$ I n most cases of polymer thin films on a substrate, higher-order elastic components had to be invoked to explain the observed spectra due to interfacial pinning, ${ }^{8}$ and hence simple liquidlike capillary fluctuations without imposed constraints due to the particular geometry have never been directly observed for polymers.

\section{Experimental Section}

Free-Standing Thin Film Preparation. To prepare thin free-standing polymer films, layers of polystyrene (PS) with the required thickness were spun onto a clean glass substrate. As listed in Table 1, PS with various molecular weights was used. The polymer radius of gyration $\left(R_{g}\right)$ in Table 1 is given by $R_{g}=6.7 \AA \times(N / 6)^{1 / 2}$ where $N$ is the polymerization index. The film thickness (d) ranged from 300 to $2500 \AA$. The films were then floated onto distilled water and deposited onto an aluminum substrate with a hole of $5 \mathrm{~mm}$ diameter at its center as shown in Figure 1. The aluminum frame outsi de of the hole was purposely roughened so that the only reflecting surface of the incident beam would be the free-standing film.

Immediately after floating the films were wrinkled, reflecting the roughness of the water surface. To relax the chains and flatten the surface after the deposition, the films were annealed in a vacuum ( $\mathrm{P} \sim 10^{-6} \mathrm{mbar}$ ). The annealing temperature was about $110^{\circ} \mathrm{C}$, which is slightly higher than the PS bulk glass transition temperature, $\mathrm{T}_{\mathrm{g}} \sim 10{ }^{\circ} \mathrm{C}$. The films were annealed above $\mathrm{T}_{\mathrm{g}}$ for $3 \mathrm{~h}$, which is much longer than a typical reptation time. Thin films annealed longer than $12 \mathrm{~h}$ occasionally developed holes. 
Table 1. Thickness and the Two Surface Roughnesses $\sigma_{1}$ and $\sigma_{2}$ Obtained from the Specular Reflectivity Data Fits

\begin{tabular}{rcccc}
\hline$d(\AA)^{\mathrm{a}}$ & $\mathrm{M}_{\mathrm{w}}(\mathrm{g} / \mathrm{mol})$ & $\sigma_{1}(\AA)^{\mathrm{b}}$ & $\sigma_{2}(\AA)^{\mathrm{b}}$ & $\mathrm{d} / \mathrm{R}_{\mathrm{g}}$ \\
\hline 494 & $1450 \times 10^{3}$ & 2.1 & 5.5 & 1.5 \\
502 & $1450 \times 10^{3}$ & 2.1 & 4.1 & 1.5 \\
450 & $2650 \times 10^{3}$ & 3.0 & 6.6 & 1.1 \\
528 & $650 \times 10^{3}$ & 3.9 & 6.5 & 2.4 \\
766 & $650 \times 10^{3}$ & 4.4 & 8.8 & 3.5 \\
1625 & $650 \times 10^{3}$ & 4.3 & 4.5 & 7.1 \\
1850 & $650 \times 10^{3}$ & 4.6 & 5.2 & 8.4 \\
760 & $284 \times 10^{3}$ & 3.8 & 5.5 & 5.2 \\
762 & $284 \times 10^{3}$ & 3.7 & 5.9 & 5.2 \\
578 & $697 \times 10^{3}$ & 3.4 & 7.3 & 2.6 \\
776 & $697 \times 10^{3}$ & 3.3 & 5.7 & 3.3 \\
957 & $697 \times 10^{3}$ & 3.8 & 5.8 & 4.2 \\
1169 & $697 \times 10^{3}$ & 3.2 & 5.2 & 5.1 \\
1631 & $697 \times 10^{3}$ & 3.5 & 4.5 & 7.1 \\
1790 & $697 \times 10^{3}$ & 4.4 & 5.8 & 7.8 \\
2272 & $697 \times 10^{3}$ & 3.7 & 4.4 & 10.0
\end{tabular}

a The error range of $d$ was $< \pm 2 \AA$. ${ }^{b}$ The uncertainties of $\sigma$ were $10-15 \%$ of the value.

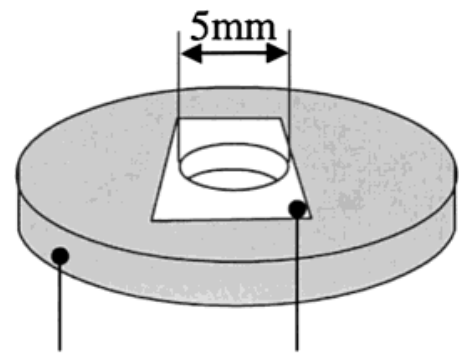

Al substrate PS film

Figure 1. Aluminum substrate with $5 \mathrm{~mm}$ hole in the center. A floated film covers the hole and aligned to the center of reflection geometry as shown in Figure 2 .

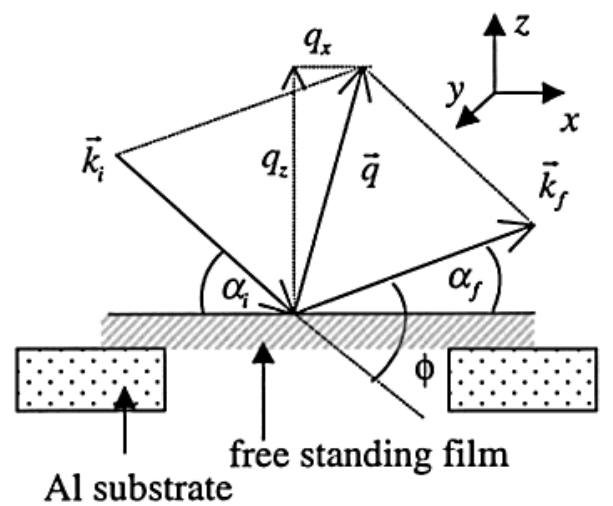

Figure 2. Scattering geometry of the $X$-ray experiments. The momentum vector transfer is defined by $\vec{q}=\vec{k}_{f}-\vec{k}_{i}$ where $\vec{k}_{i}$ and $\vec{k}_{f}$ are the incident and exit wavevector.

A specially designed sample chamber, which allows temperature control up to $200{ }^{\circ} \mathrm{C}$ under high-vacuum conditions ( $P \sim 10^{-6} \mathrm{mbar}$ ), was used for the X-ray measurements in order to reduce background scattering and protect the sample from oxidation and contamination. The chamber allows in situ X-ray measurements while varying the temperature.

X-ray Scattering. The measurements were performed at Beaml ine X10B at the National Synchrotron Light Source at Brookhaven National Laboratory using an energy of $11 \mathrm{keV}$, which corresponds to a wavelength of $\lambda=1.13 \AA$. The scattering geometry used in the experiments is shown in Figure 2. The $z, x, y$ directions are defined by the normal to the sample surface and the in-plane and out-of plane directions perpendicular to the $z$ axis, respectively. The wavevector transfer, $\vec{q}=\vec{k}_{f}-\vec{k}_{i}$ with $\vec{k}_{f}$ and $\vec{k}_{i}$ are the incident and scattered wave vectors, was controlled by varying the incident $\left(\alpha_{i}\right)$ and exit $\left(\alpha_{f}\right)$ angles with $q_{z}=k\left(\sin \alpha_{i}+\sin \alpha_{f}\right)$ and $q_{x}=$

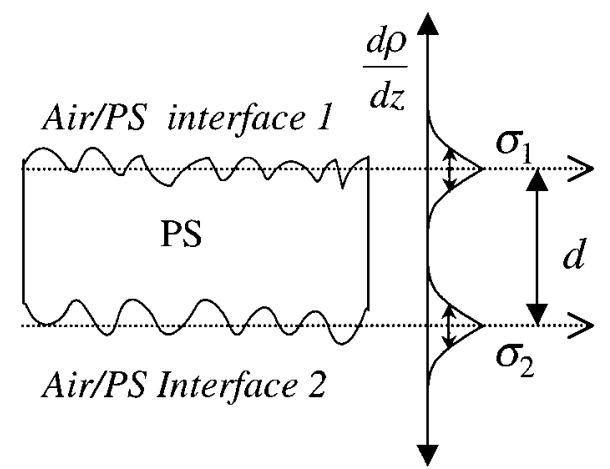

Figure 3. Sketch of a free-standing thin film with thickness, $\mathrm{d}$, and two air/polymer interfacial roughnesses, $\sigma_{1}$ and $\sigma_{2}$. The $y$-axis represents the density contrast $(d \rho / d z)$ of the free standing film schematically.

$-k\left(\cos \alpha_{i}-\cos \alpha_{f}\right)$ where $k$ is given by $k=2 \pi / \lambda$. The horizontal detector slits were wide open to achieve integration over $q_{y}$. The vertical slits were set to $0.2 \mathrm{~mm}$ defining the $\mathrm{q}_{\mathrm{x}}$ and $\mathrm{q}_{\mathrm{z}}$ resolutions.

After the X-ray measurements, the samples were examined by optical and atomic force mi croscopy to check that holes did not form during the experiment due to annealing or radiation damage. It should be noted that there is less radiation damage in free-standing films since there are no secondary electrons emitted from a substrate.

Specular Reflectivity. The specular reflectivity is defined as the intensity taken at $q_{x}=0$ as a function of $q_{z}$ by varying $\alpha_{i}$ and $\alpha_{f}$ while maintaining $\alpha_{i}=\alpha_{f}$. Since the specular reflectivity detects the variation of the el ectron density $\rho(z)$ in the direction of the surface normal, averaged in the (x,y)-plane, it is sensitive to the layer thicknesses, the density contrasts, and the interfacial roughnesses.

To analyze the reflectivity data, the recursive Parratt formalism has been used. ${ }^{19}$ The surface roughnesses $\sigma_{1}$ and $\sigma_{2}$ (see Figure 3 ) have been taken into account by modified Fresnel reflection coefficients. However, to explain the general behavior depending on the film thickness $\mathrm{d}$ and the roughnesses, the simple Born approximation is more conventional. ${ }^{20,21}$ In this approximation the reflected intensity is given by

$$
\left.I\left(q_{z}\right) \propto \frac{1}{q_{z}{ }^{4}} \int \frac{d \rho(z)}{d z} \exp \left(-i q_{z} z\right)\right|^{2}
$$

In the case of a free-standing film with mean density $\rho_{0}$, Gaussian surface roughness and sufficient large film thickness (see Figure 3),

$$
\frac{\mathrm{d} \rho}{\mathrm{d} z}=\frac{\rho_{0}}{\sqrt{2 \pi}}\left[\frac{1}{\sigma_{2}} \exp \left(-\frac{\mathrm{z}^{2}}{2 \sigma_{2}^{2}}\right)-\frac{1}{\sigma_{1}} \exp \left(-\frac{[\mathrm{z}-\mathrm{d}]^{2}}{2 \sigma_{1}^{2}}\right)\right]
$$

holds, combining eqs 1 and 2 yields

$$
\begin{aligned}
& I\left(q_{z}\right) \propto \frac{\rho_{0}{ }^{2}}{q_{z}{ }^{4}}\left[\exp \left(-q_{z}^{2} \sigma_{1}^{2}\right)\right.+\exp \left(-q_{z}^{2} \sigma_{2}^{2}\right)- \\
&\left.2 \exp \left(-q_{z}{ }^{2} \frac{\sigma_{1}{ }^{2}+\sigma_{2}{ }^{2}}{2}\right) \cos \left(q_{z} \mathrm{~d}\right)\right]
\end{aligned}
$$

Equation 3 contains damping factors due to the surface roughnesses and an oscillating term due to the interference of the scattering between both surfaces (so-called Kiessig fringes). The oscillation period $\left(\Delta q_{z}\right)$ is related to the film thickness via $\Delta \mathrm{q}_{\mathrm{z}}=2 \pi / \mathrm{d}$. If $\sigma_{1} \neq \sigma_{2}$, the term with the smaller roughness dominates the scattering for large $q_{z}$ and the oscillations due to the third term are less visible. In the case $\sigma_{1}=\sigma_{2}$, which is expected for an ideal free-standing film, eq 3 simplifies to 


$$
I\left(q_{z}\right) \propto \frac{4 \rho_{0}{ }^{2}}{q_{z}{ }^{4}} \exp \left(-q_{z}{ }^{2} \sigma_{1}{ }^{2}\right) \sin ^{2}\left(\frac{q_{z} d}{2}\right)
$$

which means that the Kiessig fringes remain visible for all $q_{z}$. Thus, the damping of the fringes directly shows whether both surfaces have identical surface roughnesses.

The goodness of fit was evaluated by a least-squares algorithm. We systematically varied and then optimized three parameters, d, $\sigma_{1}$, and $\sigma_{2}$, until $\chi^{2}$ was minimized. Since we did not find any significant improvement by varying $\rho_{0}$ as a fitting parameter, the bulk value of PS, $\rho_{0} \cong 3.37 \times 10^{-1} \mathrm{e}^{-} / \AA^{3}$ at $11 \mathrm{keV}$, has been used for the entire reflectivity fitting regardless of $\mathrm{M}_{\mathrm{w}}$ or $\mathrm{d}$.

Off-Specular Scattering. If the condition $\alpha_{i}=\alpha_{f}$ is not matched, the diffuse scattering from the sample is monitored. We again choose the Born approximation to explain the diffuse scattering of the free-standing films al though a more rigorous formalism exists (the distorted wave Born approximation, DWBA $^{20,21}$.

Compared to the specular reflectivity, which is sensitive to the density profile $\rho(z)$ perpendicular to the interfaces, the diffuse scattering depends on the interface correlation functions, which are defined as

$$
C_{i j}\left(\vec{R}_{\|}\right)=\left\langle z_{i}\left(\vec{r}_{\|}\right) z_{j}\left(\vec{r}_{\|}+\vec{R}_{\|}\right)\right\rangle
$$

where $z_{i}\left(\vec{r}_{\|}\right)$is the real space height function of the ith interface with the in-plane vector $\vec{r}_{\|}=(x, y)$.

For a free-standing film the Born approximation yields

$$
\begin{aligned}
I_{\text {diff }}(q) \propto \frac{\rho_{0}^{2}}{q_{z}^{2}}\left(e^{\left(-q_{z}^{2} \sigma_{1}^{2}\right) S_{11}\left(\vec{q}_{\| 1}\right)}+e^{\left(-q_{z}^{2} \sigma_{2}^{2}\right) S_{2}\left(\vec{q}_{\| \prime}\right)}-\right. \\
\left.2 e^{\left(-q_{z}^{2}\left(\sigma_{1}^{2}+\sigma_{2}^{2}\right) / 2\right)} \cos \left(q_{z} d\right) S_{12}\left(\vec{q}_{\|}\right)\right)
\end{aligned}
$$

with

$$
S_{i j}\left(\vec{q}_{\|}\right)=\int\left(\exp \left[q_{z}^{2} C_{i j}\left(\vec{R}_{\|}\right)\right]-1\right) \exp \left(-i \vec{q}_{\|} \vec{R}_{\|}\right) d \vec{R}_{\|}
$$

for the diffusely scattered intensity. From this equation essentially two different kinds of diffuse scattering measurements result: (1) an off-specular scan varying $q_{z}$, the so-called longitudinal diffuse scattering scan; and (2) a "constant $q_{z}$ " scan varying $\overrightarrow{\mathrm{q}}_{\|}$, which is called a transverse scan.

The longitudinal diffuse scattering scan is very similar to a specular reflectivity measurement (see eq 3 ) and shows oscillations if cross-correlations between the two interfaces are present. ${ }^{22-24}$ In particular, if the roughnesses at both surfaces are perfectly conformal, the oscillations in the diffuse scattering are in phase with the Kiessig fringes because $S_{12}\left(\vec{q}_{\| 1}\right)$ is positive. In contrast, if both surfaces are perfectly anticonformal, the diffuse scattering is out of phase with the reflectivity since $\mathrm{S}_{12}\left(\overrightarrow{\mathrm{q}}_{\|}\right)<0$.

After measuring the specular reflectivity, the longitudinal diffuse scattering intensity was recorded along $q_{z}$ while $q_{x} / q_{z}$ $=1.74 \times 10^{-3}$. The angular difference of $0.1^{\circ}$ from the secular peak was chosen in order to obtain sufficient intensity, without contamination from the intense specular peak.

Transverse scans are especially sensitive to the autocorrelation functions $\mathrm{C}_{\mathrm{ii}}\left(\overrightarrow{\mathrm{R}}_{\|}\right)$of the surfaces. ${ }^{9,21,25} \mathrm{~F}$ or a single polymer surface with cylindrical symmetry, the expected capillary wave correlation function $\mathrm{C}_{\mathrm{ii}}\left(\mathrm{R}_{\|}\right)$, can be expressed by ${ }^{9}$

$$
C_{11}(R)=\frac{B}{2}\left[K_{0}(\kappa R)-K_{0}\left(q_{u} R\right)\right]
$$

where

$$
\mathrm{B}=\mathrm{k}_{\mathrm{B}} \mathrm{T} /(\pi \gamma)
$$

the lower wavenumber cutoff is given by

$$
\kappa^{2}=\mathrm{g} \rho_{0} / \gamma+\mathrm{A}_{\mathrm{eff}} /\left(2 \pi \gamma \mathrm{d}^{4}\right)
$$

and the upper cutoff $q_{u}=1 / 6.7 \AA^{-1}$, the monomer/monomer distance given by the Kuhn length of the PS polymer. ${ }^{26}$ The symbols used are the Boltzmann constant $k_{B}$, the absolute temperature $T$, the surface tensi on $\gamma$, the accel er ation due to gravity $\mathrm{g}$, the mean density $\rho_{0}$, the film thickness $\mathrm{d}$, and the effective Hamaker constant $A_{\text {eff. }}$ The Hamaker constant takes into account the interaction with a substrate and vanishes for free-standing films. Thus, only the tiny gravitational cutoff has to be considered. The correlation function then yields the expression

$$
\sigma_{1}^{2}=\mathrm{B} / 2 \ln \left(\mathrm{q}_{\mathrm{u}} / \kappa\right)
$$

for the root-mean-square ( $\mathrm{rms}$ ) roughness of the surfaces. ${ }^{27}$ Since the gravitational cutoff $\mathrm{g} \rho \mathrm{o} / \gamma$ is on the order of $10^{-8} \AA^{-1}$, it has to be replaced by the large resolution of the experimental setup.

For in-plane wave vectors $\mathrm{q}_{||}$with a mean value larger than $\kappa$ and smaller than $q_{u}$ the correlation function of eq 6 can be well approximated by a logarithmic function with

$$
\mathrm{C}_{11}(\mathrm{R}) \approx-\mathrm{B} / 2[\ln (\kappa \mathrm{R} / 2)+0.5772]
$$

Therefore, the diffusely scattered intensity (eq 5) becomes a power law in $\mathrm{q}_{\mathrm{x}}$ (if the integration over $\mathrm{q}_{\mathrm{y}}$ due to the poor resolution of the experimental setup is already considered) with the exponent $\eta-1$ where

$$
\eta=\frac{1}{2} \mathrm{~Bq}_{\mathrm{z}}^{2}
$$

The behavior is typical for capillary waves and a fingerprint for liquidlike surfaces. ${ }^{21,25}$ This correlation formalism has been confirmed previously for simple liquid surfaces. ${ }^{9,21}$ Due to the large viscosity, it is difficult to obtain sufficiently flat polymer surfaces on substrates that are thicker than the X-ray penetration depth of a few microns. Hence, the capillary wave spectrum from a pure polymer surface without interference from substrate scattering has not yet been observed.

\section{Results and Discussion}

Figure 4 shows examples of X-ray reflectivity data (open circles) from free-standing PS films $\left(\mathrm{M}_{\mathrm{w}}=650 \times\right.$ $10^{3}$ and $697 \times 10^{3} \mathrm{~g} / \mathrm{mol}$ ) with thicknesses varying from 528 to $2275 \AA$. These thicknesses can be rescaled as ranging from $2.3 R_{g}$ to $10 R_{g}$ where $R_{g} \sim 230 \AA$ for $M_{w}=$ $697 \times 10^{3}$. The critical angle at $\mathrm{q}_{\mathrm{c}}$ of total reflection may be seen for all films. For $q_{z}<q_{c}$, the footprint of the beam was larger than the sample, and hence it was not possi ble to fit the data properly. A homogeneous single layer model with two different rms roughnesses, $\sigma_{1}$ and $\sigma_{2}$, and the corresponding error function shaped profiles on both sides of the film (see Figure 3 ) was used to fit the specular reflectivity data (solid lines in Figure 4). The quality of the fits can be seen in inset (a) of Figure 4 , showing the zoomed curves at a high- $q_{z}$ region. The fitted values for $\sigma_{1}$ and $\sigma_{2}$ and $\mathrm{d}$ are listed in Table 1 together with the parameters obtained from fits of the data taken from films of other molecular weights.

The upper curve in Figure $4(d=528 \AA)$ shows that the Kiessig fringes of the thin films are damped more rapidly compared to those of the thicker films $(d=776$, 957, 1624, and $2272 \AA$ ). As shown in Table 1, the absolute values of $\sigma_{1}$ and $\sigma_{2}$ are not significantly different from those measured on supported films; however, the asymmetry between $\sigma_{1}$ and $\sigma_{2}$ seems to increase with decreasing film thickness. This illustrates that the damping is not due to the absolute values of $\sigma$, as would be the case for the supported films, but rather 


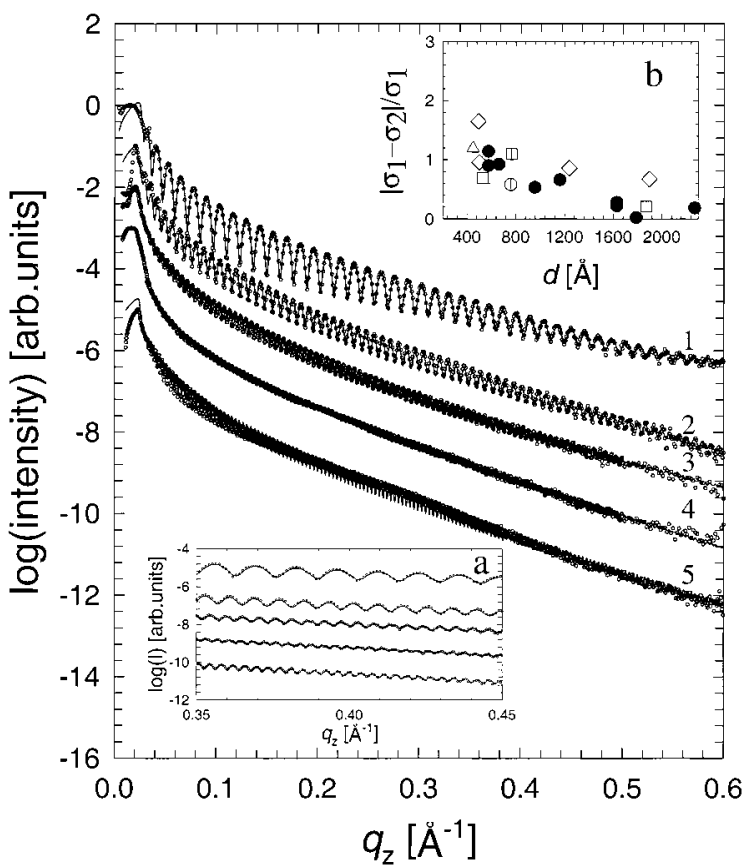

Figure 4. Measured reflectivities (symbols) and fits (solid lines) of free-standing PS films ( $1: d=528 \AA, M_{w}=650 \times$ 103; $2: d=776 \AA$; 3: $d=1168 \AA ; 4: d=1631 \AA ; 5: d=1790$ $\AA, M_{w}=697 \times 10^{3}$ for $2-5$ ) as a function of the momentum transfer $\mathrm{q}_{\mathrm{z}}$. The curves are shifted for clarity. Inset (a) displays a magnification of the region at $0.35<q_{z}\left(\AA^{-1}\right)<0.45$ to show the quality of the fits. Inset (2) shows relative differences of roughnesses from the fits as a function of film thickness $\mathrm{d}$. The mol ecular weights are denoted as follows: (O) $284 \times 10^{3}$, $(\square) 650 \times 10^{3},(\bullet) 697 \times 10^{3},(\diamond) 1450 \times 10^{3}$, and $(\triangle) 2650 \times$ $10^{3}$.

to the asymmetry between the two values as shown in eq 3. In inset (b) of Figure 4, the quantity $\Delta \sigma=\left(\mid \sigma_{1}-\right.$ $\left.\sigma_{2} \mid / \sigma_{1}\right)$ is plotted as obtained from the specular reflectivity. F rom the inset we can see that this quantity (solid circles) decreases monotonically with the film thickness, reaching zero only at thicknesses greater that $1500 \AA$. Similar reflectivity data were also obtained from films of different molecular weights. The values of $\Delta \sigma$ obtained from the respective fits are plotted as open symbols (triangles for $M_{w}=2650 \times 10^{3}$, squares for $M_{w}$ $=650 \times 10^{3}$, diamonds for $M_{w}=1450 \times 10^{3}$, and open circles for $M_{w}=284 \times 10^{3}$ ) in the figure. The large scatter observed is somewhat decreased when the thicknesses are scaled by $R_{g}$ of the polymer (Figure 5 ). However, it should be noted that $\Delta \sigma=0$ is expected for an ideal free-standing film. The fact that deviations are observed when the film thickness is less than $3 R_{g}$ indicates that the dynamics may be different in thin films.

When the films are spun-cast, the interface closest to the substrate will have a roughness that reflects the polymer/substrate interface, while the free surface will have a roughness that is a function of the surface tension corresponding to the viscous polymer solution. Once the film is floated from the substrate and annealed above $T_{g}$, the two interfaces should relax and have identical roughnesses, which are only a function of the surface tension at the annealing temperature.

At equilibrium the two surfaces of the free-standing film are completely symmetric, and there is no a priori reason for a nonvanishing value of $\Delta \sigma$. The data indicate that the relaxation process may be incomplete due to constrained dynamics when the films are thin. When

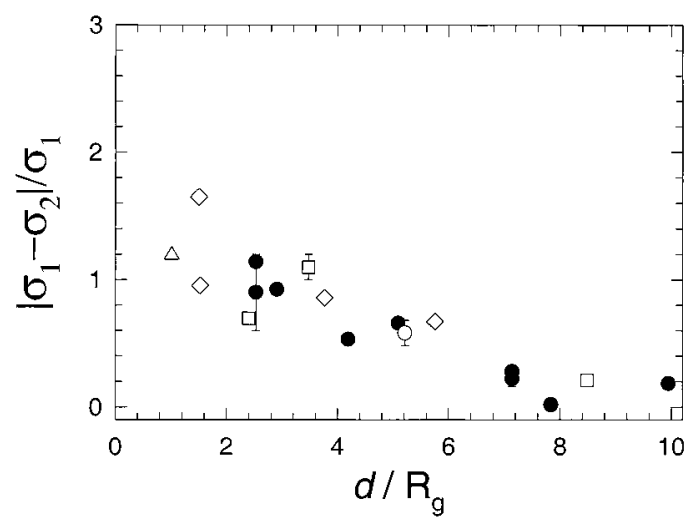

Figure 5. Difference of the roughnesses of the top surface and the bottom surface in terms of $\left|\sigma_{1}-\sigma_{2}\right| / \sigma_{1}$ vs normalized thickness $\left(\mathrm{d} / \mathrm{R}_{\mathrm{g}}\right)$. The molecular weights are denoted as follows: (○) $284 \times 10^{3},(\square) 650 \times 10^{3},(\bullet) 697 \times 10^{3},(\diamond) 1450 \times$ $10^{3}$, and $(\triangle) 2650 \times 10^{3}$.

the film thickness is on the order of a few $R_{g}$, the monomers of a single chain can span both surfaces. The relaxation dynamics at the two surfaces must therefore be correlated and can be much slower than in thick films where the two surfaces are not coupled. 28

The correlation between the roughnesses at the two interfaces in the free-standing films can also be measured directly from the longitudinal diffuse scattering. If the relaxation between the two interfaces is random, then the roughness $\sigma_{1}$ and $\sigma_{2}$ at the two surfaces should be uncorrelated. On the other hand, if the relaxation involves cooperative motion, then a correlation may build up upon annealing. Theoretically, cross-correlati ons between two surfaces should be negligible for very thick films where the chains can move independently and become considerably larger for very thin films. Specular reflectivity scans of two molecular weights ((a) $M_{w}=650 \times 10^{3}$, (b) $M_{w}=2000 \times 10^{3}$ ) for PS thin films are displayed in Figure $6 a, b$, together with the longitudinal diffuse scattering al ong $\mathrm{q}_{\mathrm{z}}$ at $\mathrm{q}_{\mathrm{x}} / \mathrm{q}_{\mathrm{z}}=1.7453 \times$ $10^{-3}\left(0.1^{\circ} \theta\right.$ off the specular direction). For clarity, each specular profile is offset by 2 decades, and the reflectivity time $\mathrm{q}_{z}{ }^{4}$ is shown. The amplitudes of the corresponding longitudinal diffuse scattering profiles are arbitrarily shifted for easier phase comparison. From Figure $6 a$ one can clearly see that for the $525 \AA$ thick film, which corresponds to a scaled thickness of one monolayer or approximately $2 R_{\mathrm{g}}$, the oscillations in the specular and longitudinal diffuse scattering profiles are completely in phase; i.e., the roughness on the surfaces is conformal. This confirms our previous assumption that the motion at the two surfaces is highly correlated. The lower trace in Figure 6a corresponds to the data of a PS film of $\mathrm{M}_{\mathrm{w}}=650 \times 10^{3}, 767 \AA$ thick or approximately $3.5 \mathrm{R}_{\mathrm{g}}$. In this case the specular and offspecular fringes are slightly out of phase, indicating that correlation may still exist. On the other hand, ref 30a has shown that an out-of-phase correlation can occur if the films have slight curvature. In this case the phase is not correlated to the dynamics. More work is in progress to differentiate the two mechanisms in this regime. A full quantitative analysis of the phase correlation for free-standing films will be given elsewhere. ${ }^{30 a, b}$

To confirm the $R_{g}$ dependence of this conformality, we studied films of different molecular weights but similar thicknesses. Figure $6 \mathrm{~b}$ shows the specular and corresponding longitudinal diffuse scattering data of PS 
(a)
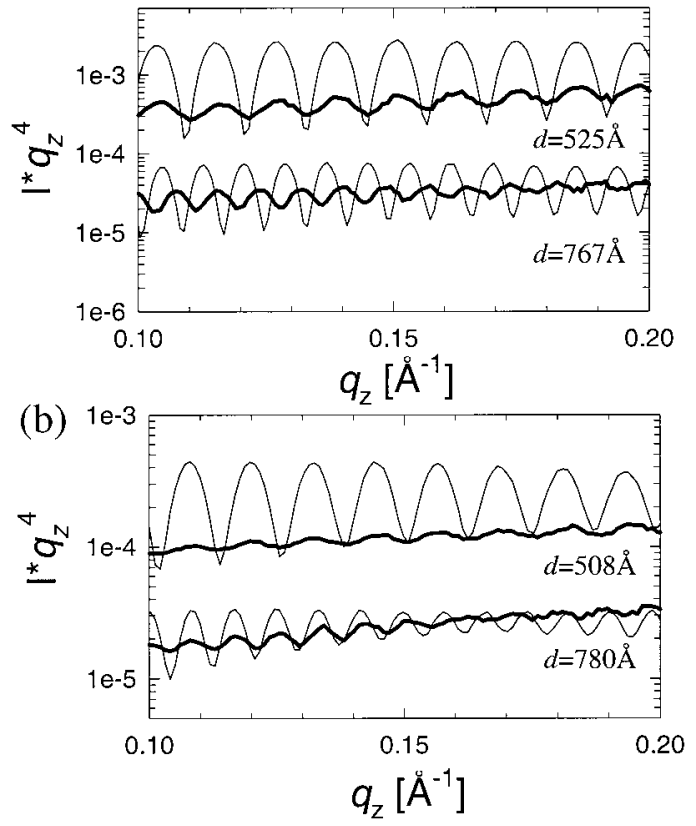

Figure 6. Specular reflectivity scans (solid lines) and the longitudinal diffuse scattering (thick solid lines) of PS freestanding films of (a) $M_{w}=650 \times 10^{3}$ and (b) $M_{w}=2000 \times 10^{3}$ al ong $q_{x} / q_{z}=1.7453 \times 10^{-3}$. The curves have been shifted for clarity.

films with $M_{w}=2000 \times 10^{3}$. From the figure we can see that, in this case, the oscillations in the longitudinal and specular scattering are in phase for both the $508 \AA$ and the $780 \AA$ films. This can be understood if we notice that for $M_{w}=2000 \times 10^{3}$ a film thickness of $780 \AA$ is still approximately $2 R_{g}$ where $R_{g} \sim 389 \AA$. Hence, the roughness of the two surfaces is still highly correlated. This shows conclusively that the degree of correlation between the two surfaces strongly depends on the molecular weight, as would be expected for film thicknesses of one polymer "monolayer".

At this point, it is interesting to compare this result with those from transverse diffuse scattering to see whether lateral capillary wave modes are also influenced by this correlation. Figure 7a depicts log-log plots of the diffusely scattered intensity of a $2240 \AA$ thick PS free-standing film of $\mathrm{M}_{\mathrm{w}}=697 \times 10^{3}$ at values at $\mathrm{q}_{\mathrm{z}}=$ $0.2,0.3$, and $0.4 \AA^{-1}$. From the figure we see that the data is well explained by a liquidlike power law decay (solid line) calculated from the correlation function given by eq 6 without any free parameters. For free-standing films the lower cutoff is simply determined by the gravitational cutoff since we do not need to consider the Hamaker constant in the expression for in eq 8. In addition, the gravitational cutoff $\mathrm{q}_{\mathrm{g}} \sim 10^{-8} \AA^{-1}$ in eq 8 is much smaller than the experimental resolution and hence does not affect the data. The exponent $\eta$ is plotted as a function of $q_{z}{ }^{2}$ in the inset. F rom the figure we can see that a good fit is obtained to a linear function, in good agreement with the predictions of the simple liquid model given by eqs 9-11. From the slope and equations, we obtain a value of the surface tension for PS of $\gamma=$ $23.8 \pm 1.2 \mathrm{mN} / \mathrm{m}$, which is lower than $\gamma=30.5 \mathrm{mN} / \mathrm{m}$ previously reported by Dee and Sauer for PS at T $=105$ ${ }^{\circ} \mathrm{C} .{ }^{31,32}$ It should be noted that the chosen $\mathrm{q}_{\mathrm{z}}$ for the transverse scans in Figure $7 b$ are not always at a maximum or a minimum in the oscillations of the longitudinal scattering as these involve the cross- (a)

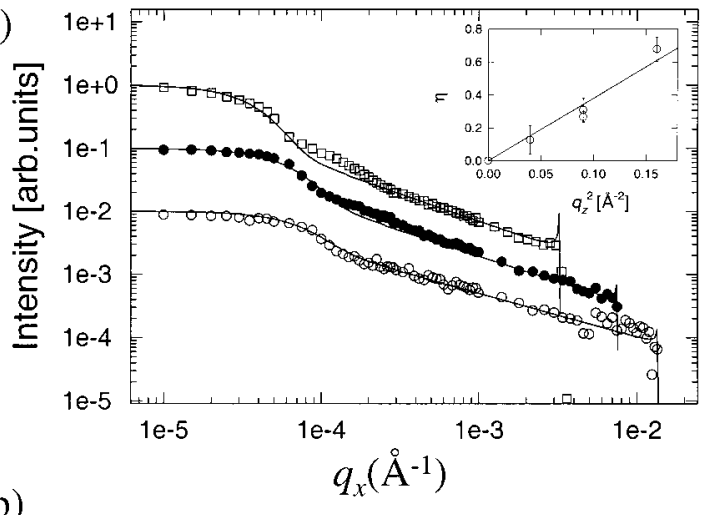

(b)

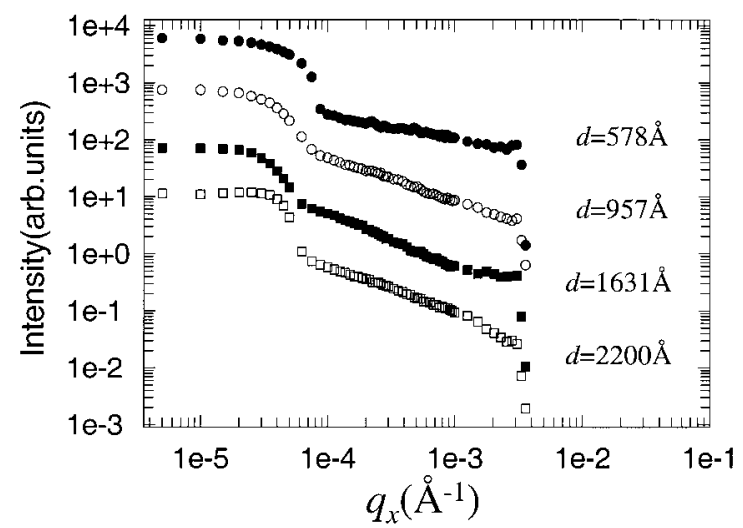

Figure 7. (a) The log-log plots of the transverse scans of a $2230 \AA$ thick PS free-standing film at $q_{z}=0.2,0.3$, and 0.4 $\AA^{-1}$. The inset shows the evolution of the exponent $\eta$ as a function of $\mathrm{q}_{z^{2}}$. (b) The log-log plots of transverse diffuse scans at $q_{z}=0.2 \AA^{-1}$ for film thickness $d=578,957,1631$, and 2200 $\AA$.

correlation functions in a different geometric way. When the film thickness is greater than $2-3 R_{g}$, the fact that an ordinary liquid capillary wave theory fits the transverse scan data so well regardless the positions of $q_{z}$ seems to indicate that the cross-correlation functions are the same as the in-plane correlation functions. In other words, the capillary waves at the two surfaces are either completely in phase or uncorrelated, and we can rule out out-of-phase correlated modes. This is in agreement with the longitudinal data which shows that, for thicknesses $3 R_{g}<d<6 R_{g}$, well-defined oscillations in the longitudinal data were present with a fixed phase factor relative to the specular (see Figure 6).

The simple liquid model can further be tested by comparing the roughness, $\sigma$, predicted from eq 9 and that obtained independently from fitting the specular reflectivity. The values of $\sigma$ obtained for different film thicknesses from the diffuse spectra are compared to those determined from the specular reflectivity in Figure 8. Since diffuse scattering cannot differentiate between the roughnesses at both surfaces and provides an average number, we compare these values to the average of $\sigma_{1}$ and $\sigma_{2}$ obtained from the specular reflectivity. Very good agreement is observed.

In Figure 7b we plot the transverse diffuse scattering for different film thickness ( $d=578,957,1631$, and $2200 \AA$ ) at $q_{\mathrm{z}}=0.2 \AA^{-1}$. F rom the figure we can see that, in contrast to supported films of the same thickness, we observe a simple power law decay in all cases without anomalous cutoffs. ${ }^{9}$ In Figure 9 we plot the surface tensions deduced from the slopes of the power law decay, as a function of film thickness. From the 


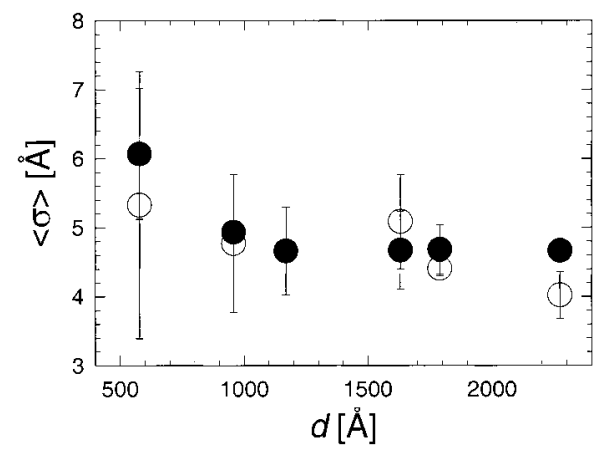

Figure 8. Average rms roughnesses fitted from specular reflectivity $(O)$ and obtained from transverse scans with eq 8 (๑) vs film thickness, d, as a function of free-standing PS film thickness, $d$.

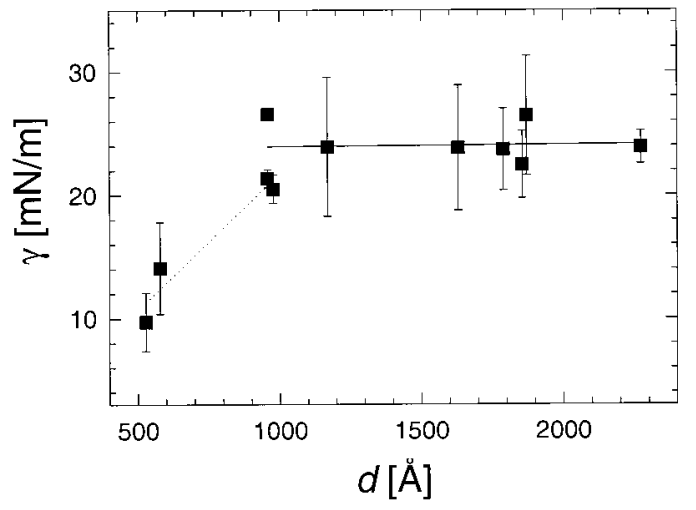

Figure 9. Surface tensions ( $\boldsymbol{\square}$ ) calculated by simple liquid model as a function of free-standing film thickness. The solid line and the dashed line are the linear fits of the data when $d$ $\geq 900 \AA$ and $d \leq 900 \AA$.

figure we can see that the surface tension is fairly constant, $\gamma=23.8 \pm 4.2 \mathrm{mN} / \mathrm{m}$, for the thick films ( $\mathrm{d}>$ $800 \AA$ ). As the films become thinner, the surface tension obtained by fitting to eq 11 decreases sharply. For the film $578 \AA$ thick, the fit yields a value of only $\gamma=14.2$ $\mathrm{mN} / \mathrm{m} \pm 3.7$, which is unrealistically low. The discontinuity in the surface tension occurs at a thickness corresponding to roughly $3 \mathrm{R}_{\mathrm{g}}$. These results indicate again that the simple liquid model can no longer be used to fit the data when $\mathrm{d}$ is on the order of a polymer monolayer. The simple liquid model assumes that all capillary waves modes are equally probable. ${ }^{21,27}$ If the modes at the two surfaces are correlated, as shown before, then only specific modes may be excited. In this case the elasticity of the film must be considered, and higher order terms than those appearing in eqs 6-11, which are proportional to the bending modulus, must be considered.

\section{Conclusion}

We have performed X-ray specular and off-specular measurements of free-standing PS thin films as a function of molecular weight and thickness. The results show that films thicker than a few monolayers are well fit by a simple liquid model. This confirms the assumption that the anomalies previously reported in the scattering intensity from polymer films of comparable thickness were mostly due to confinement by longranged interactions with the substrate rather than a fundamental property of viscoelastic fluids. The simple liquid model was found to be insufficient to fit the transverse diffuse data for films thinner than a few polymer monolayers where a monolayer corresponds to $2 R_{\mathrm{g}}$. Longitudinal diffuse data demonstrated that the roughness at the two interfaces is highly correlated when the film thicknesses were approximately $2 R_{\mathrm{g}}$. Hence, very thin films do not exhibit liquidlike behavior, and higher-order elastic terms may have to be included into models describing their rheological behavior.

Acknowledgment. This work was supported by NSF-MRSEC Program No. DMR0080604. Research carried out at NSLS (BNL), which is supported by the U.S. Department of Energy, Division of Materials Sciences and Division of Chemical Sciences. We are grate ful to Y. S. Seo, Dr. D. R. Lee, and Dr. H. J . Kim for hel pful discussions.

\section{References and Notes}

(1) J ones, R. A. L. Polymers at Surfaceand Interfaces; Cambridge University Press: Cambridge, 1998.

(2) UIman, A. An Introduction to UItrathin Organic Films from Langmuir-Blodgett to Self-Assembly; Academic Press: Boston, 1991.

(3) Liu, Y.; Russell, T. P.; Samant, M. G.; Stöhr, J .; Brown, H. R.; Cossy-Favre, A.; Diaz, J. Macromol ecules 1997, 30, 7768.

(4) Kajiyama, T.; Tanaka, K.; Takahara, A. Macromolecules 1997, 30, 280.

(5) Keddi, J . L.; J ones, R. A. L.; Cory, R. A. Europhys. Lett. 1994, 27, 59.

(6) Wallace, W. E.; van Zanten, J . H.; Wu, W. L. Phys. Rev. E 1996, 52, R3329.

(7) Zheng, X.; Sauer, B. B.; van Alsten, J. G.; Schwarz, S. A.; Rafailovich, M. H.; Sokolov, J .; Rubinstein, M. Phys. Rev. Lett. 1995, 74, 407

(8) Fredrickson, G. H.; Ajdari, A.; Leibler, L.; Carton, J .-P. Macromolecules 1992, 25, 2882.

(9) Wang, J .; Tolan, M.; Seeck; O. H.; Sinha, S. K.; Bahr; O.; Rafailovich, M. H.; Sokolov, J . Phys. Rev. Lett. 1999, 83, 564.

(10) Frank, B.; Gast, A. P.; Russell, T. P.; Brown, H. R.; Hawker C. Macromolecules 1996, 29, 6531.

(11) Overney, R. M.; Buenviaje, C.; Luginbuhl, R.; Dinelli, F. J Therm. Anal. Calorim. 2000, 59, 205.

(12) Forrest, J . A.; Dalnoki-Veress, K.; Stevens, J . R.; Dutcher, J . R. Phys. Rev. Lett. 1996, 77, 2002.

(13) Mol, E. A. L.; Wong, G. C. L.; Petit, J . M.; Rieutord, F.; de J eu, W. H. Phys. Rev. Lett. 1997, 79, 3439.

(14) Shindler, J . D.; Mol, E. A. L.; Shalaginov, A.; de J eu, W. H. Phys. Rev. Lett. 1995, 74, 722.

(15) Gierlotka, S.; Lamboy, P.; de J eu, W. H. Europhys. Lett. 1990 $12,341$.

(16) Daillant, J .; Belorgey, O. J . Chem. Phys. 1992, 97, 5824; 1992 $97,5837$.

(17) Tolan, M. X-ray Scattering from Soft-Matter Thin Films; Springer Tracts in Modern Physics; Springer: Berlin, 1999; Vol. 148.

(18) Holy, V.; Pietsch, U.; Baumbach, T. High-Resol ution X-ray Scattering from Thin Films and Multilayers; Springer Tracts in Modern Physics; Springer: Berlin, 1999; Vol. 149.

(19) Parratt, L. G. Phys. Rev. 1954, 95, 359

(20) Sinha, S. K.; Sirota, E. B.; Garoff, S.; Stanley, H. B. Phys. Rev. B 1988, 38, 2297.

(21) Sanyal, M. K.; Sinha, S. K.; Huang, K. G.; Ocko, B. M. Phys. Rev. Lett. 1991, 66, 628.

(22) (a) Garoff, S.; Sirota, E. B.; Sinha, S. K.; Stanley, H. B. J. Chem. Phys. 1989, 90, 7507. (b) Sinha, S. K. Physica B 1991 173, 25. (c) Sanyal. M. K. In Surface X-ray and Neutron Scattering; Zabel, H., Robinson, I. K., Eds.; Springer-Verlag: Berlin, 1991; p 91. (d) Schlossman, M., private communication.

(23) Tidswell, I. M.; Rabedeau, T. A.; Pershan, P. S.; Kosowsky, S. D. Phys. Rev. Lett. 1991, 66, 2108.

(24) Li, Z.; Qu, S.; Rafailovich, M. H.; Sokolov, J .; Tolan, M.; Turner, M. S.; Wang, ] . Schwarz, S. A.; Lorenz, H.; Kotthaus, J. P. Macromolecules 1997, 30, 8410.

(25) Tolan, M.; Seeck, O. H.; Schlomka, J .-P.; Press, W.; Wang, J .; Sinha, S. K.; Li, Z.; Rafailovich, M. H.; Sokolov, J. Phys. Rev. Lett. 1998, 81, 2731.

(26) Doi, M.; Edwards, S. F. The Theory of Polymer Dynamics; Clarendon Press: Oxford, 1986. 
(27) McClain, B. R.; Yoon, M.; Litster, J . D.; Mochrie, S. G. J . Eur. Phys. J. B 1999, 10, 45.

(28) The molecular weight scaling of $\Delta \sigma$ indicates that the slower dynamics are probably unrelated to $\mathrm{T}_{\mathrm{g}} . \mathrm{T}_{\mathrm{g}}$ is sensitive to local segmental relaxation and hence would be a function of absolute film thickness only rather than molecular weight. This is confirmed by direct measurements of $T_{g}$ in free standing films which found the $T_{g}$ was independent of film thickness or $M_{w}$ in the regime studies here (see ref 29).

(29) Ge, S.; Pu, Y.; Zhang, W.; Rafailovich, M.; Sokolov. J . Buenviaje, C.; Buckmaster, R.; Overney, R. M. Phys. Rev. Lett. 2000, 85, 2340.

(30) (a) Lee, D. R.; Kim, H. J .; Sinha, S. K.; Shin, K.; Rafailovich, M. H.; Sokolov, J.; Seeck, O. H.; Tolan, M., manuscript preparation. (b) The exact phase ranges from completely in- phase to $180^{\circ}$ out-of-phase and then a combination of modes as the film thickness varies from $3 R_{g}<d<5 R_{g}$ to $d<8 R_{g}$ A full calculation of the phase factor, which includes finite curvature of the films, will be presented elsewhere.

(31) Dee, G. T.; Sauer, B. B. J .Colloid Interface Sci. 1992, 152, 85.

(32) As far as we are aware, this value is closest to the bulk value for polymer thin films measured by X-ray scattering so far. Much larger deviations were found when the capillary theory was used to fit data on supported samples. The deviation from the bulk value may reflect the limitation of capillary theory when the films are very viscous and higher-order terms must be considered. For further discussion, see ref 9 .

MA001918D 